\title{
Crowdsourced disease surveillance success story: The FluWatchers program
}

\author{
Liza Lee $^{1 \star}$, Shamir Mukhi², Christina Bancej ${ }^{1}$
}

Suggested citation: Lee L, Mukhi S, Bancej C. Crowdsourced disease surveillance success story: The FluWatchers program. Can Commun Dis Rep 2021;47(9):354-6. https://doi.org/10.14745/ccdr.v47i09a01

Keywords: syndromic surveillance, crowdsourced, Canada, FluWatchers, influenza-like illness (ILI), influenza, COVID-19

\section{Introduction}

Syndromic surveillance is a core surveillance capacity for pandemic preparedness and for the detection of emerging respiratory pathogens or unexpected events related to previously circulating viruses (1). Syndromic surveillance related to illnesses such as severe acute respiratory illness and influenza-like illness (ILI) must be adaptable and ready for escalation in any pandemic (2).

Crowdsourced data collection is the process of "building a dataset with the help of a large group of people" (3). Whether you call it crowdsourcing, citizen science or participatory disease surveillance, the process of having volunteers report health information or symptoms online for the purpose of influenza surveillance is not new. Crowdsourced ILI surveillance has been in practice since 2003 and has been implemented in many countries, including Canada, because of its flexibility, low-cost, timeliness and accuracy $(4,5)$. Its use in mitigating the current coronavirus disease 2019 (COVID-19) pandemic has also been reported and assessed (6).

\section{About FluWatchers}

Since 2015, FluWatchers, the program and its current 12,000+ participants, has been contributing to the Public Health Agency of Canada's (PHAC) weekly ILI surveillance dataset and helping with the early detection of ILI activity across Canada.

FluWatchers is a participatory (crowdsourced) syndromic surveillance system that relies on Canadian volunteers to report symptoms of cough or fever to PHAC on a weekly basis. Traditional influenza surveillance systems only capture the tip of the iceberg of cases. For case information to be captured by traditional means, an individual needs to feel sick enough to seek medical care, they need to be tested, and a virus must be detected and/or isolated. FluWatchers provides a more comprehensive insight on the true burden and effects of influenza each season in the community. Traditional surveillance systems, such as laboratory surveillance, may not capture such insight because not everyone who is sick will see a doctor and, traditionally, even fewer will be tested (5). FluWatchers has been providing PHAC with reliable data on ILI activity in the community that complement the data obtained from traditional influenza surveillance sources.

The FluWatchers questionnaire is administered by and the data are managed on the Canadian Network for Public Health Intelligence (CNPHI), an established scientific public health informatics and biosurveillance platform developed and managed within PHAC's National Microbiology Laboratory. The CNPHI infrastructure provides a secure, reliable and robust technical environment for the FluWatchers program.

Volunteer participants receive a reporting link each Monday. The weekly anonymous questionnaire asks whether the participant experienced a cough or fever in the previous week and captures their vaccination status. That is it! Two quick health-related questions, 15 seconds of a participant's day and an impactful contribution to public health is made.

\section{Pivot to COVID-19 surveillance}

FluWatchers primarily collects data on symptoms, specifically cough and fever since they are typical symptoms of influenza (7). Collecting syndromic data, rather than reports of a particular disease allows the flexibility for expanded monitoring for emerging symptoms, syndromes, illnesses and self-reported diagnoses as was done with COVID-19, without sacrificing the surveillance of another disease such as influenza.

FluWatchers was able to quickly pivot to track COVID-19 in the community when the pandemic was declared. In April 2020, the FluWatchers questionnaire was quickly adapted with minimal 
changes to track COVID-19 in the community (while retaining the ability to track influenza-like illness).

The World Health Organization refers to surveillance system flexibility as the ability of a surveillance system to be adapted to meet changing needs including, but not limited to, the removal or inclusion of other diseases, modification of the reporting frequency and shifting data requirements (8). The FluWatchers program was able to include surveillance of COVID-19 by capturing information on symptoms, testing results and vaccine uptake for COVID-19 into its questionnaire. It changed its algorithm to flag a symptomatic participant from those reporting cough and fever to those reporting cough and/or fever. It also shifted to year-round surveillance from its previous reporting frame of October through May. Additionally, participation also increased from roughly 3,000 weekly participants to a high of almost 13,000 weekly participants with no negative impact on system performance. None of these inclusions required extensive changes to the existing system and as we learned more about the disease, we were able to quickly implement changes to the questionnaire. As additional work continues within the scientific community to develop appropriately sensitive and specific case definitions for COVID-19-like illness, relevant to the phases of the pandemic, FluWatchers maintains the flexibility to evolve alongside the evidence (9-13).

\section{FluWatchers' contribution to public health}

The FluWatchers program is one of two syndromic ILI surveillance programs in the national influenza surveillance system, FluWatch (14). Data collected by FluWatchers are analyzed each week and included in the FluWatch report. Data from FluWatchers are primarily used for "signal detection"-looking at the data for high or unusual influenza activity, as well as marking the start, peak and end of seasonal respiratory epidemics. The data are also published in real-time so that Canadians can see where activity is concentrated as quickly as public health officials. Access to the data is also provided on open data via Open Maps.

Since the COVID-19 pandemic, FluWatchers' data have been included in the Canada COVID-19 Weekly Epidemiology Report and have also been incorporated into COVIDTrends, a tool that provides summary data about COVID-19 in a particular area. The FluWatchers' volunteer base was recognized as a valuable source of engaged and reliable volunteers. Early in the vaccine roll-out, a time when vaccine effectiveness and safety research studies needed to be completed in a rapid fashion, the FluWatchers program was used as a means of recruiting its volunteers as participants in these studies.

Like all surveillance data, the FluWatchers' data come with their own set of limitations and biases, some of which have been amplified in the COVID-19 era $(5,10)$. There are ways to overcome or limit the effects of these biases and limitations and it really all comes down to recruiting a more diverse array of participants (such as by geography, gender, age and race) $(5,15,16)$.

\section{What is next?}

There is a solid foundation for using participatory surveillance for established and emerging disease surveillance in Canada; however, we need to build up the volunteer base prior to the circulation of the next emerging infectious disease so that when it does occur, experts can be focused on the data and not recruiting participants.

Participatory surveillance can be leveraged as governments are moving towards social innovation and open policy-making and design. FluWatchers encourages a two-way engagement between the government and its citizens, and this program can strengthen this relationship and build trust.

The number of FluWatchers participants grew over 300\% from April 2020 to April 2021 (from approximately 3,000 to 12,000+ participants). We are still not at the point where we have enough participants to reliably pick up rare signals of unusual, increased activity. There are hundreds of communities across Canada where there are only a handful of participants, and this can hamper our efforts to use FluWatchers as an elite early warning program. The more participants in an area; the more accurate the data.

If you have not already, sign-up to be a FluWatcher, spread the word to your friends, family and neighbours. In comparison, Australia's FluTracking program has over 50,000 weekly participants (17). There is nothing stopping us from reaching or exceeding that number. Let's make Canada the leader for participatory disease surveillance!

\section{Authors' statement}

$\mathrm{LL}$ - Writing, review, editing

SM - Review, editing

$\mathrm{CB}$ - Review, editing

\section{Competing interests}

None.

\section{References}

1. World Health Organization. WHO Global Epidemiological Surveillance Standards for Influenza. Geneva $(\mathrm{CH})$ : WHO; 2013. https://www.who.int/influenza/resources/ documents/WHO_Epidemiological_Influenza_Surveillance_ Standards_2014.pdf

2. Government of Canada. Surveillance Annex: Canadian Pandemic Influenza Preparedness: Planning Guidance for the Health Sector. Ottawa (ON): Government of Canada; 2015 
(accessed 2021-07-06). https://www.canada.ca/en/publichealth/services/flu-influenza/canadian-pandemic-influenzapreparedness-planning-guidance-health-sector/surveillanceannex.html

3. The World Bank. Crowd-sourced Data. World Bank; 2021 (accessed 2021-07-06). https://dimewiki.worldbank.org/ Crowd-sourced_Data

4. Smith S, Sewalk KC, Donaire F, Goodwin L, Zych A, Crawley AW, Brownstein JS, Baltrusaitis K. Maintaining User Engagement in an Infectious Disease Surveillance-Related Citizen Science Project. Citiz Sci 2021;6(1):7. DOI

5. Wójcik OP, Brownstein JS, Chunara R, Johansson MA. Public health for the people: participatory infectious disease surveillance in the digital age. Emerg Themes Epidemiol 2014;11:7. DOl PubMed

6. Leung GM, Leung K. Crowdsourcing data to mitigate epidemics. Lancet Digit Health 2020;2(4):e156-7. DOI PubMed

7. Tesini ML. Influenza In: Merck Manual. Kenilworth (NJ) USA: Merck sharp \& Dohme; 2021 (accessed 2021-07-06). https:// www.merckmanuals.com/en-ca/professional/infectiousdiseases/respiratory-viruses/influenza

8. World Health Organization. Communicable disease surveillance and response systems - Guide to monitoring and evaluating. Geneva (CH); WHO: 2006. https://www.who. int/csr/resources/publications/surveillance/WHO_CDS_EPR_ LYO_2006_2.pdf

9. Güemes A, Ray S, Aboumerhi K, Desjardins MR, Kvit A, Corrigan AE, Fries B, Shields T, Stevens RD, Curriero FC, Etienne-Cummings $\mathrm{R}$. A syndromic surveillance tool to detect anomalous clusters of COVID-19 symptoms in the United States. Sci Rep 2021;11(1):4660. DOI PubMed

10. World Health Organization. Pandemic influenza risk management: a WHO guide to inform and harmonize national and international pandemic preparedness and response. Geneva (CH): WHO; 2017. https://apps.who.int/ iris/handle/10665/259893

11. Eythorsson $E$, Helgason D, Ingvarsson RF, Bjornsson HK, Olafsdottir LB, Bjarnadottir V, Runolfsdottir HL, Bjarnadottir S, Agustsson AS, Oskarsdottir K, Thorvaldsson $\mathrm{HH}$, Kristjansdottir G, Armannsdottir B, Bjarnason A, Johannsson B, Gudlaugsson O, Gottfredsson M, Sigurdsson MI, Indridason OS, Palsson R. Clinical spectrum of coronavirus disease 2019 in Iceland: population based cohort study. BMJ 2020;371:m4529. DOI PubMed
12. Boëlle PY, Souty $C$, Launay $T$, Guerrisi $C$, Turbelin $C$, Behillil S, Enouf V, Poletto C, Lina B, van der Werf S, Lévy-Bruhl D, Colizza V, Hanslik T, Blanchon T. Excess cases of influenza-like illnesses synchronous with coronavirus disease (COVID-19) epidemic, France, March 2020. Euro Surveill 2020;25(14):2000326. DOI PubMed

13. Galli C, Pellegrinelli L, Bubba L, Primache V, Anselmi G, Delbue S, Signorini L, Binda S, Cereda D, Gramegna M, Pariani E; The Ili Sentinel Physicians Group. When the COVID-19 Pandemic Surges during Influenza Season: Lessons Learnt from the Sentinel Laboratory-Based Surveillance of Influenza-Like Illness in Lombardy during the 2019-2020 Season. Viruses 2021;13(4):695. DOI PubMed

14. Government of Canada. Overview of influenza monitoring in Canada. Ottawa (ON): Government of Canada; 2020. https://www.canada.ca/en/public-health/services/diseases/ flu-influenza/influenza-surveillance/about-fluwatch.html\#a2.3

15. Smolinski MS, Crawley AW, Olsen JM, Jayaraman T, Libel M. Participatory Disease Surveillance: Engaging Communities Directly in Reporting, Monitoring, and Responding to Health Threats. JMIR Public Health Surveill 2017;3(4):e62. DOl PubMed

16. Baltrusaitis $K$, Brownstein JS, Scarpino SV, Bakota $E_{\text {, }}$ Crawley AW, Conidi G, Gunn J, Gray J, Zink A, Santillana M. Comparison of crowd-sourced, electronic health records based, and traditional health-care based influenza-tracking systems at multiple spatial resolutions in the United States of America. BMC Infect Dis 2018;18(1):403. DOI PubMed

17. Australian Government. FluTracking. Government Australia; 2021 (accessed 2021-07-06). https://info.flutracking.net/ about/ 